\title{
Tissue Distribution of mRNA Expression of Human Cytochrome P450 Isoforms Assessed by High-Sensitivity Real-Time Reverse Transcription PCR
}

\author{
Masuhiro Nishimura, ${ }^{*, a}$ Hiroshi YAgUtI, ${ }^{a}$ Hiroki Yoshitsugu, ${ }^{a}$ \\ Shinsaku NAITO, ${ }^{a}$ and Tetsuo SATOH ${ }^{b}$ \\ Department of Drug Metabolism, Division of Pharmacology, Drug Safety and Metabolism, Otsuka \\ Pharmaceutical Factory, Inc., ${ }^{a}$ Naruto, Tokushima 772-8601, Japan, and Non-Profit Organization \\ Human \& Anima Bridging Research Organization, ${ }^{b}$ Shiroi, Inba, Chiba 270-1402, Japan
}

(Received December 4, 2002; Accepted February 20, 2003)

\begin{abstract}
Pairs of forward and reverse primers and TaqMan probes specific to each human cytochrome P450 isoform were prepared. Analysis of the mRNA level of each CYP isoform in total RNA from pooled specimens of various human organs was performed by real-time reverse transcription PCR using an ABI PRISM 7700 sequence detector system. The expression of CYP3A4 mRNA was similar to that of CYP3A7 mRNA in the fetal liver, and CYP3A4 mRNA levels in the fetal liver were about 0.1 times lower than in the adult liver. CYP2E1 showed the highest level of mRNA expression in the liver. The mRNA expression of $30 \mathrm{CYP}$ isoforms (CYP1A1, 1A2, 1B1, 2A6, 2A7, 2B6, 2C8, 2C9, 2C18, 2C19, 2D6, $2 \mathrm{E} 1,2 \mathrm{~J} 2,3 \mathrm{~A} 4,3 \mathrm{~A} 5,3 \mathrm{~A} 7,4 \mathrm{~A} 11,4 \mathrm{~F} 2,4 \mathrm{~F} 3,5 \mathrm{~A} 1,7 \mathrm{~B} 1,8 \mathrm{~A} 1,8 \mathrm{~B} 1,17,26 \mathrm{~A} 1,27,27 \mathrm{~B} 1,39 \mathrm{~A} 1,46$, and 51) in the liver was successfully detected by this method. CYP2F1, 4B1, 4F8, 11s (11A, 11B1, and 11B2), 19, and $24 \mathrm{mRNA}$ levels were the highest in the lung, lung, prostate, adrenal gland, placenta, and kidney, respectively; however, the mRNA expression of these eight CYP isoforms in the liver was not detected by this method. The mRNA levels of the CYP isoforms determined in various human tissues were in good agreement with previously reported data. The method described here has the advantages of high specificity and excellent quantification over a wide range of mRNA concentrations, making it suitable for the evaluation of a large number of samples in the assessment of the expression profile of drug-metabolizing enzymes.
\end{abstract}

Key words_ cytochrome P450; quantification; real-time RT-PCR; TaqMan probe

\section{INTRODUCTION}

The human cytochrome P450 system consists of a number of CYP isoforms that are of vital importance for the termination of pharmacologic and toxic effects. ${ }^{1,2)}$ Many CYP families such as CYP1, CYP2, CYP3, CYP4, CYP5, CYP7, CYP8, CYP11, and CYP17 are found in humans, and the main site of CYP expression is the liver. ${ }^{2)}$ It has been thought that xenobiotics are mainly metabolized by the CYP families CYP1, CYP2, and CYP3, suggesting that endogenous substrates are biotransformed by the other families. $^{3)}$

It is difficult to determine the activity levels of these CYP isoforms in the same sample because a large amount of sample is required for measurement. In order to evaluate the expression of CYP isoforms, the Western blot method and the Northern blot method are widely employed for the determination of CYP content and mRNA content, respectively. Vari-

e-mail: nisimums@otsukakj.co.jp ous procedures for the determination of CYP molecular mRNA content have been reported to date, ${ }^{4,5)}$ but these are not suitable for high-throughput measurement of samples because they are very time-consuming processes. Recently, the mouse liver6) and cultured human hepatocytes ${ }^{7)}$ have been reported to provide higher sensitivity in the determination of CYP isoform mRNA content using reverse transcription PCR (RT-PCR).

The present study was therefore undertaken to investigate the tissue distribution of the mRNA expression of 38 human CYP isoforms using a highthroughput, semiautomated, single-tube RT-PCR assay method.

\section{MATERIALS AND METHODS}

\section{Materials}

Pooled total RNA prepared from adult human tissues (liver, small intestine, kidney, adrenal gland, lung, brain, prostate gland, testis, uterus, and placenta) was purchased from CLONTECH Laboratories Inc. (Hilden, Palo Alto, CA, USA) for use in this 
Table 1. Total RNA Source Information and Values for Human $\beta$-Actin and P450 mRNAs in Various Tissues

\begin{tabular}{|c|c|c|c|c|c|c|c|c|c|c|c|}
\hline CYP & Iiver & Fetal liver & $\begin{array}{c}\text { Small in- } \\
\text { testine }\end{array}$ & Kidney & $\begin{array}{l}\text { Adrenal } \\
\text { gland }\end{array}$ & Lung & Brain & Prostate & Testis & Uterus & Placenta \\
\hline Pool size & 2 & 63 & 11 & 8 & 67 & 5 & 1 & 47 & 19 & 10 & 3 \\
\hline Age & 15,35 & $\begin{array}{l}23-40 \\
\text { weeks }\end{array}$ & $15-60$ & $24-55$ & $17-72$ & $14-40$ & 28 & $14-50$ & $17-61$ & $15-77$ & $23-31$ \\
\hline Sex & $\mathrm{F}, \mathrm{M}$ & $\mathrm{F}, \mathrm{M}$ & $\mathrm{F}, \mathrm{M}$ & $\mathrm{F}, \mathrm{M}$ & $\mathrm{F}, \mathrm{M}$ & $\mathrm{F}, \mathrm{M}$ & M & M & M & $\mathrm{F}$ & $\mathrm{F}$ \\
\hline Race & $\mathrm{C}$ & $\mathrm{C}$ & C & C & C & $\mathrm{C}$ & A & $\mathrm{C}$ & $\mathrm{C}$ & $\mathrm{C}$ & C \\
\hline $\begin{array}{l}\text { Cause of } \\
\text { death }\end{array}$ & $\begin{array}{l}\text { sudden } \\
\text { death }\end{array}$ & $\begin{array}{l}\text { spon- } \\
\text { taneous } \\
\text { abortion }\end{array}$ & trauma & trauma & $\begin{array}{l}\text { sudden } \\
\text { death }\end{array}$ & $\begin{array}{l}\text { sudden } \\
\text { death }\end{array}$ & $\begin{array}{l}\text { sudden } \\
\text { death }\end{array}$ & $\begin{array}{l}\text { sudden } \\
\text { death }\end{array}$ & trauma & trauma & no death \\
\hline$\beta$-actin & 0.0832 & 0.0678 & 0.331 & 0.0733 & 0.249 & 0.561 & 0.0848 & 0.275 & 0.103 & 0.394 & 0.337 \\
\hline CYP1A1 & 0.0594 & 0.000272 & 0.00176 & 0.000239 & 0.00677 & 0.0679 & 0.000388 & .00465 & 0.00215 & 0.0201 & 0.00101 \\
\hline CYP1A2 & 4.77 & BLQ & BLQ & 0.000021 & 0.00113 & 0.000119 & BLQ & 0.000740 & 0.00141 & 0.00193 & BLQ \\
\hline CYP1B1 & 0.00578 & 0.000622 & 0.0128 & 0.0139 & 0.0144 & 0.0167 & 0.00174 & 0.104 & 0.0197 & 0.0413 & 0.00490 \\
\hline СYР2A6/7 & 27.8 & 0.199 & 0.00193 & 0.00188 & 0.0105 & 0.0622 & 0.00867 & 0.00809 & 0.0187 & 0.0390 & 0.0231 \\
\hline CYP2A6 & 27.5 & 0.184 & BLQ & BLQ & BLQ & & 0.00731 & BLQ & BLQ & 0.00447 & 0.000611 \\
\hline CYP2A7 & 0.617 & 0.00908 & 0.00249 & BLQ & 0.000322 & 0.000063 & 0.000619 & 0.000646 & 0.000600 & 0.00295 & 0.00131 \\
\hline CYP2B6 & 1.46 & 0.0675 & 0.0537 & 0.0797 & 0.00178 & 0.456 & 0.00162 & 0.00558 & 0.00485 & 0.00796 & 0.000920 \\
\hline CYP2C8 & 10.2 & 0.226 & 0.0166 & 0.00660 & 0.0364 & 0.00481 & 0.00214 & 0.00197 & 0.0720 & 0.000444 & BLQ \\
\hline CYP2C9 & 3.11 & 0.0245 & 0.188 & 0.00295 & 0.000493 & 0.000334 & 0.000622 & 0.000111 & 0.000072 & 0.000592 & 0.000149 \\
\hline CYP2C18 & 5.31 & 0.0188 & 1.41 & 0.00174 & BLQ & 0.0122 & 0.00148 & BLQ & 0.0240 & 0.0197 & 0.00425 \\
\hline CYP2C19 & 0.187 & 0.000152 & 0.0303 & 0.0000148 & 0.000030 & 0.000038 & 0.000014 & 0.000001 & 0.000033 & 0.000037 & 0.000015 \\
\hline CYP2D6 & & & & & & & & & & & \\
\hline CYP2E1 & 53.8 & 0.419 & & & & & & & & & \\
\hline CYP2F1 & BLQ & BLQ & BLQ & BLQ & BLQ & 0.0128 & BLQ & BLQ & 0.000124 & BLQ & BLQ \\
\hline CYP2J2 & 0.319 & 0.0759 & 0.0517 & 0.00832 & 0.00203 & & & & 0.0201 & 0.00408 & 0.0556 \\
\hline СУР3А3/4 & 3.07 & & & & & & & & & & \\
\hline CYP3A4 & & & & & & & & & 0.00101 & & 0.000094 \\
\hline CYP3A5 & 0.220 & 0.0120 & 0.0624 & 0.0195 & 0.0226 & 0.0188 & 0.000485 & 0.0232 & BLQ & 0.00303 & 0.000517 \\
\hline CYP3A7 & 0.0226 & 0.268 & BLQ & 0.00515 & BLQ & 0.000872 & 0.00603 & 0.00196 & BLQ & BLQ & BLQ \\
\hline CYP4A11 & 4.56 & & 0.0440 & & & & & & & & 0.000335 \\
\hline & & & & & & & & & & & 0.0188 \\
\hline CYP4F2 & 1.26 & 0.00989 & 0.0775 & 0.315 & 0.000030 & 0.00160 & 0.000754 & 0.0348 & 0.00751 & 0.000022 & BLQ \\
\hline CYP4F3 & 1.14 & 0.205 & 0.0728 & 0.217 & 0.000730 & 0.0140 & 0.00739 & 0.00263 & 0.00267 & 0.000177 & 0.00918 \\
\hline & & & 0.000051 & & 0.000943 & & & & & & \\
\hline CYP5A1 & & & & & & & & & & 0.0520 & 0.0740 \\
\hline CYP7B1 & 0.415 & 0.0346 & 0.0711 & & & & 0.0375 & & 0.0921 & 0.115 & 0.0352 \\
\hline CYP8A1 & 0.00110 & 0.00101 & 0.00380 & 0.000670 & 0.00404 & 0.00991 & 0.000161 & 0.0152 & 0.0125 & 0.0686 & 0.00100 \\
\hline CYP8B1 & 3.45 & 0.582 & 0.000352 & & 0.000275 & 0.000254 & & & 0.000708 & & 0.0349 \\
\hline CYP11A & & 0.00144 & BLQ & & 16.8 & & & 0.00142 & 0.732 & 0.00255 & 8.90 \\
\hline CYP11B1 & & & & & & & & & BLQ & BLQ & BLQ \\
\hline CYP11B2 & BLQ & BLQ & BLQ & BLQ & 0.871 & BLQ & BLQ & BLQ & BLQ & BLQ & BLQ \\
\hline CYP17 & 0.0345 & 0.00883 & BLQ & 0.127 & 76.6 & & & & 1.18 & 0.000901 & 0.000667 \\
\hline CYP19 & BLQ & 0.0890 & 0.000718 & BLQ & 0.0246 & 0.000552 & 0.00192 & 0.00545 & 0.0173 & 0.00206 & 26.0 \\
\hline CYP24 & BLQ & BLQ & BLQ & 2.64 & BLQ & 0.0686 & 0.00168 & 0.0413 & 0.000295 & 0.0795 & 0.0248 \\
\hline CYP26A1 & 0.0405 & 0.000228 & BLQ & BLQ & 0.00575 & 0.000740 & 0.00529 & BLQ & 0.00353 & 0.00760 & 0.0109 \\
\hline CYP27 & 1.13 & 0.175 & 0.0909 & 0.119 & & & 0.0158 & 0.112 & & & 0.00599 \\
\hline CYP27B1 & 0.00560 & 0.00104 & 0.0115 & 0.183 & 0.0262 & 0.00746 & 0.000578 & 0.00658 & 0.0137 & 0.00321 & 0.00407 \\
\hline CYP39A1 & 0.875 & 0.0527 & 0.0251 & 0.0465 & 0.0377 & 0.0576 & 0.00317 & 0.257 & 0.129 & 0.0439 & 0.00398 \\
\hline CYP46 & 0.00763 & 0.000526 & 0.00315 & 0.00630 & 0.00749 & 0.00346 & 0.162 & 0.00617 & 0.0253 & 0.0145 & 0.000349 \\
\hline CYP51 & 1.61 & 0.989 & 0.340 & 0.236 & 1.84 & 0.710 & 0.286 & 1.29 & 2.36 & 0.328 & 0.205 \\
\hline
\end{tabular}

BLQ, below the limit of quantification, F; female, M; male, C; Caucasian, A; Asian. Data are expressed as the ratio of $\beta$-actin or CYP mRNA to GAPDH mRNA. Experiments were performed in triplicate. 
study. In addition to these adult human tissues, total RNA prepared from fetal liver was also purchased from CLONTECH Laboratories Inc. Yeast tRNA was purchased from Life Technologies, Inc. (Rockville, MD, USA), and the TaqMan One-Step RTPCR Master Mix Reagents Kit, TaqMan GAPDH Control Reagents (Part No. 402869), TaqMan $\beta$-Actin Control Reagents (Part No. 401846), Micro Amp $^{\circledR}$ Optical 96-Well Reaction Plates, Optical Adhesive Covers, and Optical Cover Compression Pads were all purchased from Applied Biosystems (Foster City, CA, USA). All other chemicals used in this study were of reagent grade.

\section{Methods}

2.1 Oligonucleotides The pairs of forward and reverse primers and the TaqMan probes for CYP1A1, CYP1A2, CYP1B1, CYP2A6, CYP2B6, CYP2C8, CYP2C9, CYP2C18, CYP2C19, CYP2D6, CYP2E1, CYP2J2, CYP3A4, CYP3A5, CYP4A11, and CYP27 used for the RT-PCR employed sequences that have been reported previously. ${ }^{8)}$ The pairs of primers and the TaqMan probes for the other CYP mRNAs were designed using Primer Express software (Applied Biosystems). The nucleotide positions of the oligonucleotides were as follows.

CYP2A6/7 (GenBank accession numbers AF182275 and NM_000764)

forward primer (position from the initiation codon: 173-193) :

5'-TCATGAAGATCAGTGAGCGCT-3'

reverse primer (254-236):

5'-TCATGTCCACACAGCACCA-3'

probe (195-218):

5'-TGGCCCCGTGTTCACCATTCACTT-3'

CYP2A7 (GenBank accession number NM_000764)

forward primer (861-884):

5'-CTTGAAGAACCTGATGATGAGCAC-3'

reverse primer (1000-978):

5'-CTCTGTCAATCTCCTCATGGACC-3'

probe (889-913):

5'-AACCTCTTCATTGCAGGCACCGAGA-3'

CYP2F1 (GenBank accession number NM_000774)

forward primer (585-606):

5'-GCTCACCATTATCCGCCTTATC-3'

reverse primer (744-723):

5'-GAGGTCTCTCAGGCACTTGAAG-3'

probe (608-635):

5'-ATGACAACTTCCAAATCATGAGCAGCC
C-3

CYP3A3/4 (GenBank accession numbers

NM_000776 and AF182273)

forward primer (850-872):

5'-ACTGAGTCCCACAAAGCTCTGTC-3'

reverse primer (1014-993):

5'-AACTGCATCAATTTCCTCCTGC-3'

probe $(876-905)$ :

5'-TCTGGAGCTCGTGGCCCAATCAATTATC TT- $3^{\prime}$

CYP3A7 (GenBank accession number NM_000765)

forward primer (684-705):

5'-CCTTACCCCAATTCTTGAAGCA-3'

reverse primer (881-859):

5'-TCCAGATCAGACAGAGCTTTGTG-3'

probe (850-819):

5'-AGTCTTTTGAATTCTGAGAGTCAATCAT-

CAGC-3'.

CYP4B1 (GenBank accession number NM_000779)

forward primer (974-995):

5'-CCTGGTTTCTCTACTGCATGGC-3'

reverse primer (1081-1061):

5'-CCAGATCATCCCACTGGAAGA-3'

probe (997-1024):

5'-CTGTACCCTGAGCACCAGCATCGTTGTA$3^{\prime}$

CYP4F2 (GenBank accession number NM_001082)

forward primer (1092-1113):

5'-CCGTGAGCCTAAAGAGATTGAA-3'

reverse primer (1276-1256):

5'-CGAAAACACTGATGAGGCAGA-3'

probe (1182-1205):

5'-AGTCCCGGTCATCTCCCGCCATGT-3'

CYP4F3 (GenBank accession number NM_000896)

forward primer (1073-1094):

5'-TACAAGAGCTTCTGAAGGACCG-3'

reverse primer (1267-1246):

5'-TGATGAGGCAGATAATGCCTTT-3'

probe (1188-1212):

5'-TGCCGTCTCTCGCTGCTGCACCCAA-3'

CYP4F8 (GenBank accession number NM_007253)

forward primer (1278-1299):

5'-AATCCATCACAACCCCTCAGTC-3'

reverse primer (1390-1371):

5' -CCGCCGAGAAAGGAATAAAA-3'

probe (1341-1370):

5'-CGAAAACGCCCAGAAGAGGTCACCTAT

GGC-3'

CYP5A1 (GenBank accession number M80647) 
forward primer (291-313):

5'-CAAGCAGGTGTTGGTTGAGAACT-3'

reverse primer (370-350):

5'-TGTCGGCTACCGACTTGAACT-3'

probe (320-347):

$5^{\prime}$-ACTTTACCAACAGAATGGCGTCGGGTTT-

$3^{\prime}$

CYP7B1 (GenBank accession number NM_004820)

forward primer $(800-820)$ :

5'-GGCAAGATGTCCTGGAGAAAT- ${ }^{\prime}$

reverse primer (935-915):

5'-GGGTGCCGCAGAAGATAATAC-3'

probe $(822-851): 5^{\prime}$-TTATGTGCACGAGGACC

TTGAAATAGGAGC- $3^{\prime}$

CYP8A1 (GenBank accession number NM_000961)

forward primer (166-186):

5'-AGGATGAAGGAGAAGCACGGT-3'

reverse primer (313-293):

5'-CCATGAGGAAGATGGCATAGG-3'

probe (217-243):

5'-TATGTCACCGTTCTCCTGGACCCACAC-3'

CYP8B1 (GenBank accession number NM_004391)

forward primer (585-607):

5'-CCTGCTACAGGCAGGAGAGTTAT- ${ }^{\prime}$

reverse primer (659-636):

57-AGGGAGTAGACAAACCTTGGGAAA-3'

probe (609-636):

5'-CATGGAGTTCCGCAAGTTTGACCTTCTT-

$3^{\prime}$

CYP11A (GenBank accession number NM_000781)

forward primer (223-244):

5'-CACCTTCACCATGTCCAGAATT-3'

reverse primer (305-284):

5'-ATGACATAAACCGACTCCACGT-3'

probe (246-272):

5'-CCAGAAGTATGGCCCGATTTACAGGGA$3^{\prime}$

CYP11B1 (GenBank accession number NM_000497)

forward primer (1158-1179):

5'-GAGCTCAGACTTGGTGCTTCAG-3'

reverse primer (1415-1396):

5'-TGGAGGTGTTTCAGCACATG-3'

probe (1209-1234):

5'-GCGCGTGTTCCTCTACTCTCTGGGTC-3'

CYP11B2 (GenBank accession number NM_000498)

forward primer (1167-1189):

5'-CTTGGTGCTTCAGAACTACCACA-3'

reverse primer (1429-1407):

5'-TTAGTGTCTCCACCAGGAAGTGC-3' probe (1209-1239):

5'-ACAGGTTTTCCTCTACTCGCTGGGTCGC

AAT-3'

CYP17 (GenBank accession number NM_000102)

forward primer (1200-1221):

5'-TCACAATGAGAAGGAGTGGCAC-3'

reverse primer (1294-1272):

5'-AGCTTACTGACGGTGAGATGAGC-3'

probe (1230-1255):

5'-TCAGTTCATGCCTGAGCGTTTCTTGA-3'

CYP19 (GenBank accession number NM_000103)

forward primer (414-435):

5'-AGAGCTCTGGAAAACAACTCGA-3'

reverse primer (487-467):

5'-CTGTGACCATACGAACAAGGC-3'

probe (437-465):

5'-CCTTCTTTATGAAAGCTCTGTCAGGCCC

C-3'

CYP24 (GenBank accession number XM_030592)

forward primer (732-752):

5'-AATGATGAGCACGTTTGGGAG-3'

reverse primer (806-788):

5'-TGCCAGACCTTGGTGTTGA-3'

probe (754-779):

5'-ATGATGGTCACTCCAGTCGAGCTGCA-3'

CYP26A1 (GenBank accession number XM_051144)

forward primer (884-904):

5'-TTCGACTGAATCCCCCAGTTC-3'

reverse primer (1022-1002):

5'-TTGGTGAAGATCTCTGCCACA-3'

probe (909-939):

5'-AGGGTTTCGGGTTGCTCTGAAGACTTTT

GAA-3'

CYP27B1 (GenBank accession number XM_048549)

forward primer (1349-1367):

5'-TTGGCAAGCGCAGCTGTAT-3'

reverse primer (1423-1403):

5'-GTGTTAGGATCTGGGCCAAAG-3'

probe (1374-1399):

5'-ACGCCTGGCAGAGCTTGAATTGCAAA-3'

CYP39A1 (GenBank accession number NM_016593)

forward primer (101-121):

5'-GCTGGATTCCTTGGATTGGAG-3'

reverse primer (219-200):

5'-CATTCGGTTTCCCATAGCAA-3'

probe (123-148):

5'-TGGATTTGAGTTTGGGAAAGCCCCTC-3'

CYP46 (GenBank accession number NM_006668)

forward primer (1126-1146): 


\section{5'-GAGGAGACCTTGATTGATGGG-3'}

reverse primer (1228-1208):

5'-TCAGCGGGTCCTCAAAGTATG-3'

probe (1167-1192):

5'-CCCGCTCTTGTTCAGCACCTATGTCA-3'

CYP51 (GenBank accession number XM_029186)

forward primer (409-429):

5'-TACAGTCGCCTGACAACACCT-3'

reverse primer (518-498):

5'-TGGGCTATGTTAAGGCCACTT- ${ }^{\prime}$

probe (434-460):

5'-TTGGGAAGGGAGTTGCATACGATGTGC$3^{\prime}$

The primer and/or probe was homology searched by an NCBI BLAST search to ensure that it was specific for the target mRNA transcript. The primers and TaqMan probes were synthesized by Sawady Technology Co., Ltd. (Tokyo, Japan). The TaqMan probes contained 6-carboxyfluorescein (FAM) at the $5^{\prime}$ end and 6-carboxytetramethylrhodamine (TAMRA) at the $3^{\prime}$ end and were designed to hybridize to a sequence located between the PCR primers.

2.2 Experimental Conditions for TaqMan RTPCR The total RNAs obtained from fetal human liver and adult human tissues (liver, small intestine, kidney, adrenal gland, lung, brain, prostate gland, testis, uterus, and placenta) were diluted with water to $20 \mu \mathrm{g} / \mathrm{ml}$ in the absence of RNase, then further diluted with $50-\mu \mathrm{g} / \mathrm{ml}$ yeast tRNA. To prepare the calibration curve, various amounts of $50-\mu \mathrm{g} / \mathrm{ml}$ yeast tRNA ranging from 1.28 to $100000 \mathrm{pg}$ total RNA were used. For the RT-PCR reaction, the TaqMan One-Step RT-PCR Master Mix Reagents Kit (Applied Biosystems) containing $300 \mathrm{nM}$ forward primer, $900 \mathrm{nM}$ reverse primer, and $200 \mathrm{nM}$ TaqMan probe was used at $50 \mu \mathrm{l} /$ tube. Amplification and detection were performed using the ABI PRISM 7700 Sequence Detector System (Applied Biosystems) with the following profile: 1 cycle at $48^{\circ} \mathrm{C}$ for $30 \mathrm{~min}, 1 \mathrm{cy}$ cle at $95^{\circ} \mathrm{C}$ for $10 \mathrm{~min}$, and 40 cycles each at $95^{\circ} \mathrm{C}$ for $15 \mathrm{sec}$ and $60^{\circ} \mathrm{C}$ for $1 \mathrm{~min}$. For GAPDH, $200 \mathrm{nM}$ forward primer, $200 \mathrm{nM}$ reverse primer, and $100 \mathrm{nM}$ TaqMan probe were used, and for $\beta$-actin, $300 \mathrm{nM}$ forward primer, $300 \mathrm{nM}$ reverse primer, and $200 \mathrm{nM}$ TaqMan probe were used.

2.3 Data Analysis Samples were deemed positive at any given cycle when the value of the emitted fluorescence was greater than the threshold value cal- culated by the instrument's software (Sequence Detector Ver. 1.6.3). The threshold cycle (Ct), which is defined as the cycle at which PCR amplification reaches a significant value (i.e., usually 15 times the standard deviation of the baseline), is given as the mean value. The relative expression of each mRNA was calculated by the $\Delta \mathrm{Ct}$ (the value obtained by subtracting the $\mathrm{Ct}$ value of GAPDH mRNA from the $\mathrm{Ct}$ value of the target mRNA) method. Specifically, the amount of target mRNA relative to GAPDH mRNA was expressed as $2^{-(\Delta \mathrm{Ct})}$. Data are expressed as the ratio of target mRNA to GAPDH mRNA. Studies were conducted in triplicate and data are shown as mean values.

\section{RESULTS AND DISCUSSION}

Analysis was conducted by RT-PCR using the ABI PRISM 7700 Sequence Detector System in the presence of the TaqMan probe. For preparation of the CYP calibration curves, the total RNA obtained from the small intestine (CYP1B1), lung (CYP2F1, CYP4B1), fetal liver (CYP3A7), prostate gland (CYP4F8), uterus (CYP8A1), adrenal gland (CYP11A, CYP11B1, CYP11B2, CYP17), placenta (CYP19), kidney (CYP24, CYP27B1), and brain (CYP46) was used for the individual CYP isoforms. The source of total RNA for the other CYP isoforms was the adult human liver. The lower limit of quantification of each of the mRNAs ranged from $1.28 \mathrm{pg}$ to $800 \mathrm{pg}$ of total RNA per $50 \mu \mathrm{l}$ of reaction mixture (data not shown). It has been suggested that the analytical sensitivity of the TaqMan RT-PCR assay is 10 -100 times greater than that of conventional nested RT-PCR. ${ }^{9)}$ No post-amplification steps are required, resulting in very simple processing and a short assay time. In addition, the assay can be performed in a high-throughput manner by using 96-well or 384-well plates.

Table 1 shows the total RNA source information and values for human $\beta$-actin and P450 mRNAs in various tissues. GAPDH was used as the endogenous control in the measurement of target mRNAs. The $\beta$ actin/GAPDH ratio differed less than 10 times among all target tissues. All CYP isoforms except for CYP11B1 and CYP11B2 showed a tissue-specific expression pattern. It was clearly recognized that the mRNA content of the 38 CYP isoforms could be determined using the method described in this report. For the CYP3A subfamily, CYP3A4 showed the 
highest level of mRNA expression and CYP3A5 showed the second highest level of mRNA expression in the adult liver, while CYP3A7 showed the highest level of mRNA expression and CYP3A4 showed a level of mRNA expression similar to that of CYP3A7 in the fetal liver. Consistent with the findings of another study, ${ }^{10)}$ CYP3A4 mRNA levels in the fetal liver were about 0.1 times lower than in the adult liver. The results for the CYP3A subfamily in the fetal liver and in adult organs such as the liver, small intestine, kidney, and lung are also consistent with the data reported by Raunio et al.,11) Zhang et al.,12) Lacroix et al., ${ }^{10)}$ and De Wildt et al. ${ }^{13)}$ CYP2E1 showed the highest level of mRNA expression in the adult liver, and consistent with the findings of other studies, ${ }^{14,15)}$ CYP2E1 mRNA levels were about 20 times higher than those of CYP3A4 in the adult liver. CYP2A6 showed the second highest level of mRNA expression in the adult liver. The expression pattern for the CYP2C subfamily in the adult liver in this study was similar to previously reported data for the CYP2C subfamily. ${ }^{16)}$ Furthermore, consistent with the findings of other studies, ${ }^{17,18)}$ CYP2C9 mRNA levels were 17 times higher than CYP2C19 mRNA levels in the adult liver. CYP2D6 mRNA levels were about 0.25 times lower than CYP3A4 mRNA levels in the adult liver, similar to the findings reported by Rodriguez-Antona et al. ${ }^{18)}$ The mRNA expression of 30 CYP isoforms (CYP1A1, 1A2, 1B1, 2A6, 2A7, 2B6, 2C8, 2C9, 2C18, 2C19, 2D6, 2E1, 2J2, 3A4, $3 \mathrm{~A} 5,3 \mathrm{~A} 7,4 \mathrm{~A} 11,4 \mathrm{~F} 2,4 \mathrm{~F} 3,5 \mathrm{~A} 1,7 \mathrm{~B} 1,8 \mathrm{~A} 1,8 \mathrm{~B} 1,17$, $26 \mathrm{~A} 1,27,27 \mathrm{~B} 1,39 \mathrm{~A} 1,46$, and 51) in the liver was successfully detected by this method. CYP2F1, 4B1, $4 \mathrm{~F} 8,11 \mathrm{~s}$ (11A, 11B1, and 11B2), 19, and 24 mRNA levels were the highest in the lung, lung, prostate, adrenal gland, placenta, and kidney, respectively; however, the mRNA expression of these eight CYP isoforms in the adult liver was not detected by this method. CYP1A1 and CYP1A2 mRNA levels were the highest in the adult lung and liver, respectively. The CYP1A1 mRNA level was 570 times higher than the CYP1A2 mRNA level in the lung, while that of CYP1A2 was 80 times higher than that of CYP1A1 in the adult liver. The results for CYP1A1 and CYP1A2 mRNA in the small intestine, lung, and placenta were similar to those reported in the literature. ${ }^{11,12,19)}$ The results for CYP2B6, CYP2F1, and CYP4B1 mRNA in the lung were also similar to those reported in the literature. ${ }^{11)}$ CYP1A2, CYP2F1, and CYP4B1 were absent in the fetal liver, which is in agreement with the literature. ${ }^{20,21)}$ In addition, the mRNA expression profiles in these cases showed no overlap among molecular species. Although the specificity for the expression of the mRNAs of CYP11B1 and 11B2 was verified only for the adrenal gland, the sequences of the primers and the probe were specified based on a homology study by performing an NCBI BLAST search. In summary, the mRNAs of 38 cytochrome $\mathrm{P} 450$ isoforms were determined differentially using the TaqMan one-step RT-PCR method.

In conclusion, the method described here has a number of significant advantages such as high sensitivity, simplicity, and quantification over a wide range of mRNA concentrations. This method is suitable for the evaluation of a large number of test samples in the assessment of the expression profiles of drug-metabolizing enzymes.

\section{REFERENCES}

1) Gonzalez F. J., Pharmacol. Ther., 45, 1-38 (1990).

2) Hakkola J., Pelkonen O., Pasanen M., Raunio H., Crit. Rev. Toxicol., 28, 35-72 (1998).

3) Gonzalez F. J., Trends Pharmacol. Sci., 13, 346-352 (1992).

4) Mattes W. B., Li A. P., Chem. Biol. Interact., 107, 47-61 (1997).

5) Finnström N., Bjelfman C., Thörn M., LööF L., Rane A., J. Lab. Clin. Med., 134, 133-140 (1999).

6) Pan J., Xiang Q., Ball S., Drug Metab. Dispos., 28, 709-713 (2000).

7) Bowen W. P., Carey J. E., Miah A., McMurray H. F., Munday P. W., James R. S., Coleman R. A., Brown A. M., Drug Metab. Dispos., 28, 781-788 (2000).

8) Nishimura M., Yoshitsugu H., Naito S., Hiraoka I., Yakugaku Zasshi, 122, 339-361 (2002).

9) Gut M., Leutenegger C. M., Huder J. B., Pedersen N. C., Lutz H., J. Virol. Methods, 77, 37-46 (1999).

10) Lacroix D., Sonnier M., Moncion A., Cheron G., Cresteil T., Eur. J. Biochem., 247, 625634 (1997).

11) Raunio H., Hakkola J., Hukkanen J., Lassila A., Päivärinta K., Pelkonen O., Anttila S., Piipari R., Boobis A., Edwards R. J., Exp. 
Toxic. Pathol., 51, 412-417 (1999).

12) Zhang Q. Y., Dunbar D., Ostrowska A., Zeisloft S., Yang J., Kaminsky L. S., Drug Metab. Disp., 27, 804-809 (1999) .

13) De Wildt S. N., Kearns G. L., Leeder S., van den Anker J. N., Clin. Pharmacokinet., 37, 485-505 (1999).

14) Sumida A., Kinoshita K., Fukuda T., Matsuda H., Yamamoto I., Inaba T., Azuma J., Biochem. Biophys. Res. Commun., 262, 499503 (1999).

15) Andersen M. R., Farin F. M., Omiecinski C. J., DNA Cell Biol., 17, 231-238 (1998).

16) Gerbal-Chaloin S., Pascussi J. M., PichardGarcia L., Daujat M., Waechter F., Fabre J. M., Carrere N., Maurel P., Drug Metab. Dispos., 29, 242-251 (2001).
17) Rodríguez-Antona C., Donato M. T., Pareja E., Gómez-Lechón M. J., Castell J. V., Arch. Biochem. Biophys., 393, 308-315 (2001).

18) Rodríguez-Antona C., Jover R., GómezLechón M. J., Castell J. V., Arch. Biochem. Biophys., 376, 109-116 (2000) .

19) Hakkola J., Pasanen M., Hukkanen J., Pelkonen O., Mäenpää J., Edwards R. J., Boobis A. R., Raunio H., Biochem. Pharmacol., 51, 403-411 (1996).

20) Hakkola J., Pasanen M., Purkunen R., Saarikoski S., Pelkonen O., Mäenpää J., Rane A., Raunio H., Biochem. Pharmacol., 48, 5964 (1994).

21) Ring J. A., Ghabrial H., Ching M. S., Smallwood R. A., Morgan D. J., Pharmacol. Ther., 84, 429-445 (1999). 